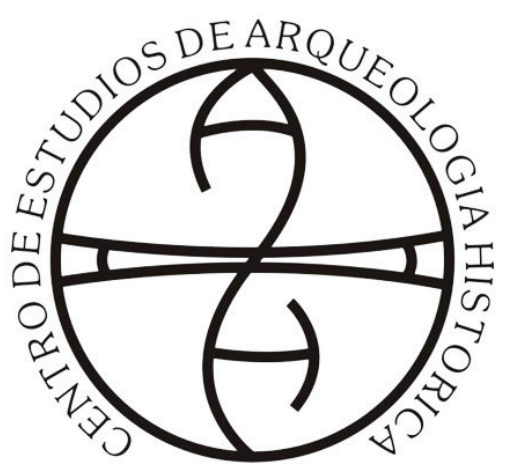

Centro de Estudios de Arqueología Histórica

Universidad Nacional de Rosario

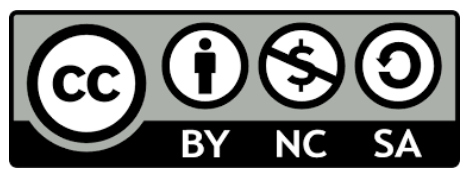

Teoría y Práctica de la Arqueología Histórica Latinoamericana | Año X, Volumen 13 | 2021

Revista del Centro de Estudios de Arqueología Histórica, Facultad de Humanidades y Artes,

Universidad Nacional de Rosario https://teoriaypracticaah.unr.edu.ar/index.php/index https://rephip.unr.edu.ar/handle/2133/14804

ISSN en línea: 2591-2801

ISSN versión impresa: 2250-866X

Atribución-NoComercial-CompartirIgual 4.0 Internacional (CC BY-NC-SA 4.0)

https://creativecommons.org/licenses/by-nc-sa/4.0/deed.es

Camila Oliva (ID: https://orcid.org/0000-0002-3907-7457), Guillermo Bertani (ID: https://orcid.org/0000-0002-88791286) y María Carlota Sempé (ID: https://orcid.org/00000001-7466-3235). Redes de aprovisionamiento y memorias descriptivas de las construcciones funerarias del patrimonio histórico de la ciudad de La Plata

\title{
REDES DE APROVISIONAMIENTO Y MEMORIAS DESCRIPTIVAS DE LAS CONSTRUCCIONES FUNERARIAS DEL PATRIMONIO HISTÓRICO de la ciudad de La Plata
}

\section{Provisioning NETWORKS AND DESCRIPTIVE MEMORIES OF THE FUNERAL CONSTRUCTIONS OF THE HISTORICAL HERITAGE FROM THE LA Plata CITY}

\author{
Camila Oliva *, Guillermo Bertani ** y María Carlota Sempé ***
}

\section{Resumen}

El día 19 de noviembre de 1882 Pedro Benoit, secundado por los Maestros mayores de obra de la Provincia de Buenos Aires construyeron un arco de bóveda subterráneo y cubrieron la piedra Fundamental-procedente de Azul- con ladrillos y mezcla utilizando una cuchara de oro macizo con ocho brillantes en la empuñadura de ébano y una batea para la mezcla de caoba con agarraderas de ébano e incrustaciones doradas. Este acontecimiento inauguró la etapa de construcción de la ciudad de La Plata.

El proceso de urbanización de la nueva capital de la provincia de Buenos Aires, se caracterizó por un rápido desenvolvimiento y dinamismo, acompañado de un crecimiento demográfico explosivo. En este

* Laboratorio de Análisis Cerámico UNLP. Argentina. oliva.cami@gmail.com

** Laboratorio de Análisis Cerámico UNLP. Argentina. gobertani@gmail.com

*** Laboratorio de Análisis Cerámico UNLP. Argentina. carlota_sempe@yahoo.com.ar 
marco, se desplegó una importante red comercial caracterizada por el flujo de materiales constructivos tanto a nivel nacional como internacional. Este trabajo se propone dar cuenta de los circuitos comerciales de aprovisionamiento de las materias primas utilizadas en la construcción durante la etapa fundacional de la ciudad de La Plata entre fines del siglo XIX y principios del XX. Metodológicamente, se trabajó tanto con fuentes arqueológicas como documentales, usándose la información procedente de los expedientes de obras particulares correspondientes al Cementerio de La Plata, que posibilitaban recortar más estrictamente la etapa fundacional. En este sentido, se analizaron diversos contextos mortuorios (bóvedas) de esta primera etapa de la necrópolis de la ciudad, conjugando esta información con la proporcionada por los catálogos de arte funerario cívico y religioso disponibles para la época, los cuales constituyen una importante fuente documental patrimonial.

Palabras clave: fundación de La Plata; aprovisionamiento materia primas; patrimonio funerario; urbanización; redes comerciales.

\begin{abstract}
On November 19, 1882, Pedro Benoit, seconded by the Senior Construction Masters of the Province of Buenos Aires, built a vault arch underground and covered the Foundation stone - brought from Azul with bricks and mix using a solid gold spoon with eight brilliants on the ebony handle and a mahogany mix pan with ebony handles and gold inlays. This event inaugurated the construction stage of the city of La Plata. The urbanization process of the new capital of the province of Buenos Aires was characterized by rapid development and dynamism, accompanied by explosive demographic growth. Within this framework, an important commercial network was deployed characterized by the flow of construction materials both nationally and internationally. This work aims to account for the commercial circuits for the supply of raw materials used in construction during the founding stage of the of La Plata city between the end of the 19th century and the beginning of the 20th.

Methodologically, we worked with both archaeological and documentary sources, using the information from the records of private works corresponding to the La Plata Cemetery, which made it possible to cut the founding stage more strictly. In this sense, various mortuary contexts (vaults) of this first stage of the city's necropolis were analyzed, combining this information with that provided by the catalogs of civic and religious funerary art available for the time, which constitute an important patrimonial documentary source.
\end{abstract}

Keywords: La Plata foundation; supply of raw materials; funeral heritage; urbanization; commercial networks.

\title{
Introducción
}

Este trabajo tiene por objeto presentar los resultados del estudio de los circuitos productivos y comerciales del aprovisionamiento de materias primas utilizadas en la construcción edilicia durante la etapa fundacional de la Ciudad de La Plata, entre fines del siglo XIX y principios del XX. Los mismos fueron reconstruidos y analizados a partir del estudio de una muestra de construcciones funerarias erigidas en dicho período.

Tal objetivo se sustenta en la premisa fundante del presente trabajo, que considera al patrimonio funerario como un testimonio privilegiado de los valores construidos por una sociedad en una época y por sus costumbres. Asimismo, forma parte de los procesos de construcción histórica y de la identidad 
cultural de una comunidad dada. Por lo cual, entendemos que investigar, es también una forma de patrimonializar el pasado, tanto en su materialidad como en su intangibilidad, porque nos remite a los modos en que los agentes sociales de ese pasado significaban y pensaban. Son esos aspectos en los que concordamos con Fava (2019) cuando sostiene que: "Ya sea individual o colectivo, el patrimonio, como capital de nuestros orígenes, nunca puede ser reducido al sólo 'haber'. Ser otro y autorizar a otro en el tiempo, ese es el dinamismo del auténtico proceso patrimonial” (p.9).

\section{Proceso fundacional de la Ciudad de La Plata}

Considerando que un proyecto de Gran Escala se caracteriza por su corto desarrollo temporal, gigantismo del emprendimiento y el aislamiento (Ribeiro, 1987), es que concordamos con el planteo de Catullo quien considera la fundación de la ciudad de La Plata como el primer Proyecto de Gran Escala de la República Argentina (Catullo, 1998; 2015). La elección del lugar de asentamiento de la nueva capital provincial, aseguró su aislamiento, porque en 1881, el partido de Ensenada, distrito escogido para la fundación, era una zona poco habitada, con una población urbano-rural absoluta de 6962 personas. Su sede urbana era un caserío de 414 casas habitadas por 358 familias, según el censo realizado durante el gobierno de Rocha (Censo General Provincia de Buenos Aires, 1883).

En cuanto a su temporalidad, luego del acto fundacional el 19 de noviembre de 1882, el proceso de urbanización platense fue acelerado. En 1884, en solo dos años, la ciudad poseía sus principales edificios públicos, escuelas y más de 1000 viviendas en el ejido urbano (Coni, 1885). Igualmente, tenía una red caminera de $2000 \mathrm{~km}$, que conectaba la capital con las localidades del interior y, las autoridades provinciales estaban definitivamente asentadas. Respecto al Gigantismo, el ejido urbano constaba de $25 \mathrm{~km}^{2}$, dentro del cual habitaban en ese momento 10.407 personas (Barba, 1998). En este núcleo poblacional el $78 \%$ era extranjero, representando un crecimiento demográfico explosivo para la época y la región por el flujo inmigratorio de los contratos de obreros y profesionales en Europa. En este marco, el crecimiento demográfico continuó y en 1909 alcanzó los 95.126 habitantes (Salas y Condomi Alcorta, 1910).

Por su parte, el proceso constructivo de los edificios públicos y viviendas implicó un flujo importante de materiales de construcción de Argentina y Uruguay. Entre otros puede mencionarse al granito empleado en el empedrado de calles, veredas, construcción de edificios y obras viales.

\section{EI Cementerio de La Plata}

El Cementerio de la Ciudad de La Plata, fue inaugurado el primero de febrero de 1887 mediante un decreto del Poder Ejecutivo Provincial. Se hallaba ubicado en el sector sureste de la urbe por afuera de la misma. Su plano, características y arquitectura neoclásica fueron proyectados por el ingeniero Pedro Benoit -al igual que el plano de la ciudad y de muchos de los edificios públicos- este espacio funerario se creó respondiendo a los mismos simbolismos. Se puede afirmar que la ciudad de los vivos tiene su reflejo en la ciudad de los muertos, subdividido en cuatro secciones principales (Figura 1). En la época fundacional contaba con $29.960 \mathrm{~m}^{2}$, abarcando un total de 16 manzanas. Actualmente, su sector principal se encuentra ocupado por panteones sociales y familiares de características monumentales y gran riqueza ornamental, totalizando unas 2500 estructuras. De acuerdo al censo platense publicado en 1910 (op. cit, 1910), existían unas 46 construcciones funerarias, más las inhumaciones realizadas en la galería de nichos ubicadas en los laterales de la entrada principal (Figura 1).

A los fines de este trabajo hemos analizado aquellas correspondientes a la etapa ocupacional más 
antigua (panteones del año 1902 a 1920). De estos panteones seleccionamos una muestra representativa de las memorias constructivas por razones de espacio e ilustración, a la que sumamos otros casos publicados por integrantes del proyecto (Carrasquero, Adam y Sempé, 2012).
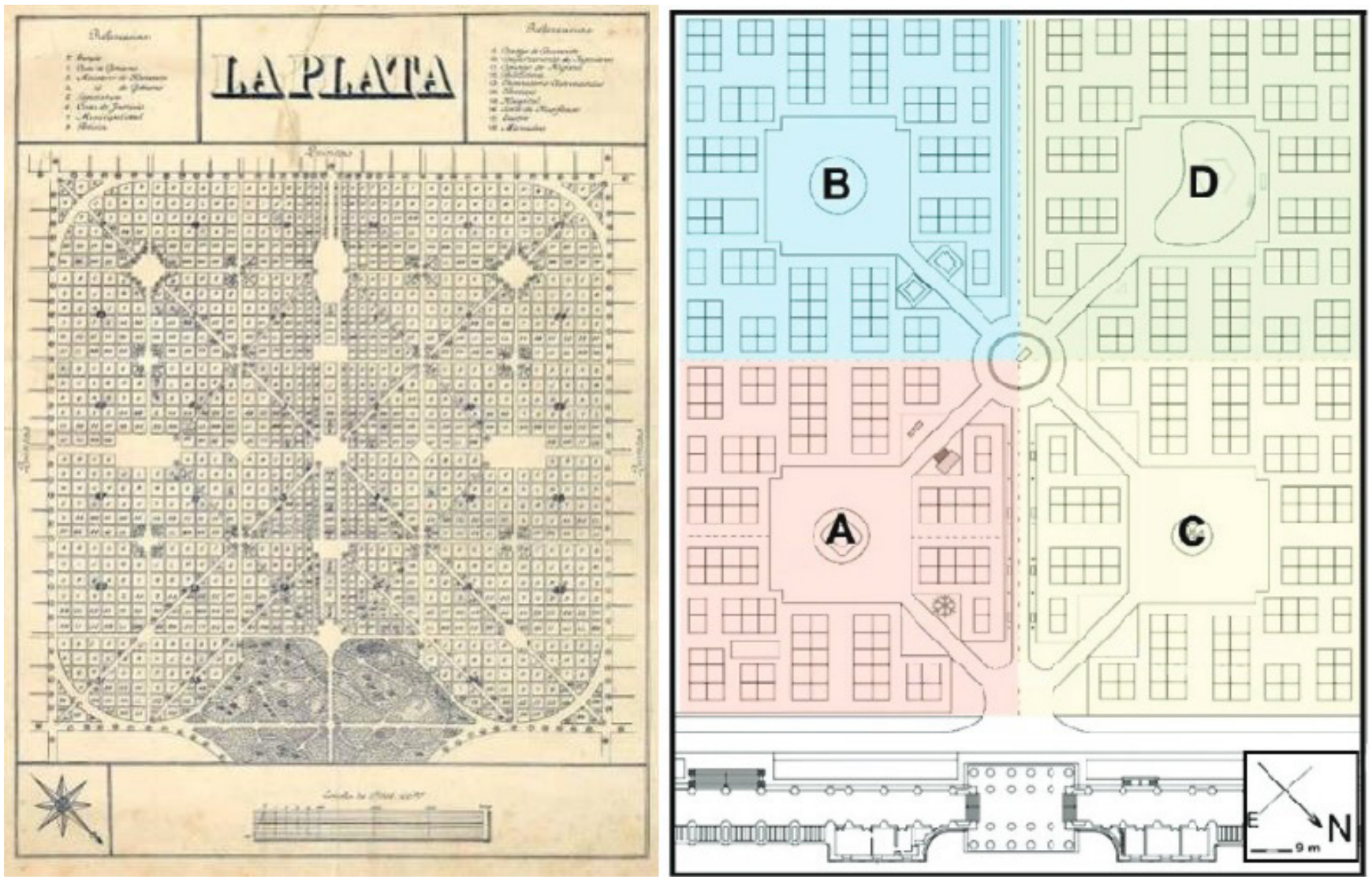

Figura 1. Izquierda: Plano histórico de la Ciudad de La Plata. Derecha: Plano del Cementerio de La Plata. Se observa la correspondencia entre ambos planos. Fuente: izquierda Archivo General de la Nación, Mapoteca II-117; derecha modificado de Delledonne, Masola y Corbalán, 2011.

\section{Materiales y métodos}

Una de las tareas iniciales de esta investigación fue el relevamiento fotográfico de las construcciones funerarias del Cementerio de La Plata. Posteriormente, se completó este registro con la información documental existente en diversos archivos, como los planos de construcción de panteones familiares y sociales de la Oficina de Obras particulares de la Municipalidad, cuyos expedientes logramos fueran trasladados a las oficinas del Cementerio, a fin de que en ellas estuvieran reunidos todos los archivos históricos pertinentes.

En el archivo del Museo Dardo Rocha se consultaron los avisos publicados en la Guía Mensual Revista La Plata de los años 1885-1887 relacionados a materiales de construcción, los comerciantes del ramo, constructores y profesionales arquitectos e ingenieros residentes en la ciudad, así como la consulta 
de bibliografía especializada publicada, en especial sobre la geología de la provincia de Buenos Aires.

\section{Información brindada por las memorias descriptivas de las bóvedas.}

A los fines aquí propuestos, se analizaron los expedientes del archivo de obras particulares, sección cementerio correspondientes a la etapa fundacional. Éstos cuentan con información sobre los materiales de construcción usados en los panteones familiares y de las asociaciones de época. En el presente trabajo ejemplificamos con información de diferentes panteones cuyas memorias constructivas fueron examinadas y cotejadas con su respectivo panteón en el cementerio.

El primer caso lo constituye el Panteón Logiudice (Figura 2a), ubicado en el lote 26 sección A del Cementerio de La Plata. (Municipalidad de La Plata (MPL), Oficina técnica, trámites internos, 1908. Permiso de construcción Panteón A 26), La Memoria descriptiva fue elaborada por Carlos Villar y expresa que:

Los ladrillos serán de $1^{\text {a }}$ calidad, bien cocidos y de buen sonido. La mezcla se usará de la forma siguiente, para la mampostería 1 parte de cal del Azul. 2 de arena del río, y 1 de polvo de ladrillo, para el reboque interno la misma mezcla reforzada con portland, el reboque exterior será de imitación piedra. La pared del sótano y piso llevaran cemento hidrófugo.

Otra construcción que da cuenta de los materiales utilizados son los del panteón de Rómulo Merlo (Figura 2b). (Municipalidad de La Plata (MLP), Oficina técnica, trámites internos, 1908. Permiso de construcción Panteón A 52). En este sentido puede leerse que:

Los ladrillos serán de los comunes de cal bien cocidos y de buen sonido (...) se empleará una de cal del Azul en pasta, dos de arena del río de La Plata y una de polvo de ladrillo. Para el reboque (sic) se empleará la mezcla de una de cal del Azul en pasta, dos de arena del río de La Plata, una de arena Oriental y una de polvo de ladrillo. En las molduras, además de la mezcla anterior se fratachará con cal fina y Portland. Capa aisladora en la pared del sótano: se aplicará una capa de cemento hidrófugo encima de la que se colocará el reboque (sic). (...) El hormigón para el piso del sótano será hecho de 5 partes de pedregullo, tres partes de arena Oriental y de una parte de Portland (...) la mezcla será de 2 partes de cal del Azul apagada en piletas y una parte de arena vizcaína y 4 partes de arena del río. Para las vigas de fierro se colocarán piedras de granito para su descanso.

Otro caso es el panteón Marcellini, ubicado en el Lote 67 de la sección A (Figura 2c). En su memoria descriptiva puede leerse que “(...) 2 partes de cal del Azul, 2 de arena del río, 2 de polvo de ladrillo 2 de arena oriental (...) En los frentes se empleará la mescla (sic) de 2 partes de arena oriental 1 de portland y 1 de tierra romana amarilla." (Municipalidad de La Plata, Oficina técnica, trámites internos, 1907. Permiso de construcción Panteón A 67)

Igualmente, se analizó la memoria constructiva del Panteón 15-24 ubicada también en la sección A. (Municipalidad de La Plata, Oficina técnica, trámites internos, 1902. Permiso de construcción Panteón A 15-24). Ésta corresponde al sepulcro de Guillermo Tunessi (Figura 2d) y la memoria fue firmada por Carlos Glade y Carlos Pedemonte, la información obtenida fue que "Las paredes del sótano serán hechas 
con ladrillos comunes y argamasa formada con cal del Azul y conchilla. En las paredes superiores los revoques exteriores e interiores serán en símil piedra formados con Portland, tierra romana en poca parte y arena oriental". El siguiente caso considerado es el Panteón 40 sección A, correspondiente a la bóveda de Joaquín Sese (Figura 2e), en cuya memoria constructiva realizada por Enrique Madrid con fecha 16 de marzo de 1906 registra el uso de:

(...) ladrillos de cal de $1^{\mathrm{a}}$ calidad bien cocidos y de buen sonido. Las mesclas se usarán en la proporción siguiente 1 parte cal del Azul, 1 de arena del río de La Plata y 2 de polvo de ladrillo. (...) El reboque (sic) del frente una imitación piedra. (Municipalidad de La Plata, Oficina técnica, trámites internos, 1906. Permiso de construcción Panteón A 40)

Fue analizado igualmente, el Panteón 22 localizado en la sección A, correspondiente a Parodi (Figura 2 f). Su memoria constructiva data del año 1902 y declara los materiales que debían emplearse para su edificación (Municipalidad de La Plata, Oficina técnica, trámites internos, 1902. Permiso de construcción Panteón A 22):

Los ladrillos serán de $1^{\text {a }}$ calidad bien cocidos y de buen sonido. La mescla a emplearse para la mampostería será en la proporción de una parte de cal del Azul dos partes de arena del río de La Plata y una parte de polvo de ladrillo, para el reboque (sic) se empleará la mescla una parte de cal una de arena del río una de polvo de ladrillo, una de arena oriental y media de Portland

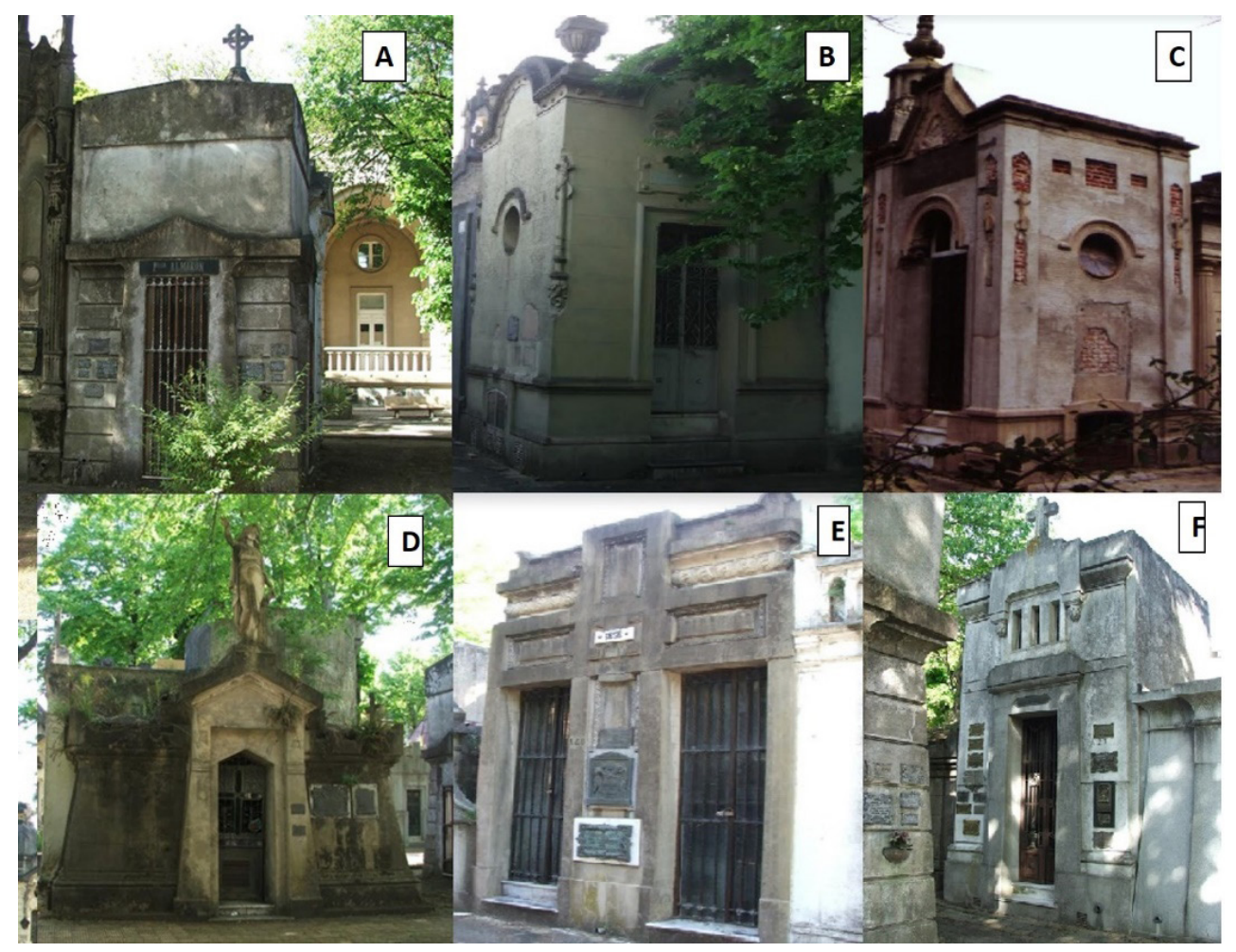

Figura 2. En orden sucesivo de izquierda a derecha, los panteones Logiudice (a), Rómulo Merlo (b), Marcelini (c), Tunessi (d), Sesse (e) y Parodi (f). 
De acuerdo a trabajos previos (Carrasquero et al., 2012) en el sector de panteones y nicheras del Cementerio, varias sepulturas presentan trabajos en mármol de procedencia italiana como los denominados carrara, botticino, rosado y travertino entre otros. En relación a este tema se consideraron dos casos de estudio. Por un lado, el de Domingo Lastra, ubicado en la Sección C lote 95 y data del año 1904 (Figura 3a). Constituido por un hipogeo (sepultura subterránea) cubierto en superficie con una columna truncada apoyada sobre una base escalonada, donde se colocó la tapa de entrada a la cámara subterránea, que lleva la inscripción "A Domingo Lastra 1904" y más abajo "Rosa Hamilton de Lastra 1935". El conjunto está rodeado por cadenas sostenidas por cuatro pequeñas columnas. El material usado es el mármol blanco de Carrara.

Finalmente, se consideró la sepultura con monumento de Carlos Jaureguy, ubicada en la sección D lotes 67-68 y data del año 1906 (Figura 3b). Se halla contra la pared lateral de la bóveda familiar Jaureguy-Navajas (D 67-68), su tapa abovedada lleva la inscripción "A Carlos Jaureguy. Sus amigos" y una foto esmaltada del occiso.

En la cabecera, se levanta un importante pedestal sobre el cual se apoya el busto del personaje y a nivel de base, en los laterales detalles fitomorfos tallados. Todo el monumento está realizado en mármol blanco de carrara.

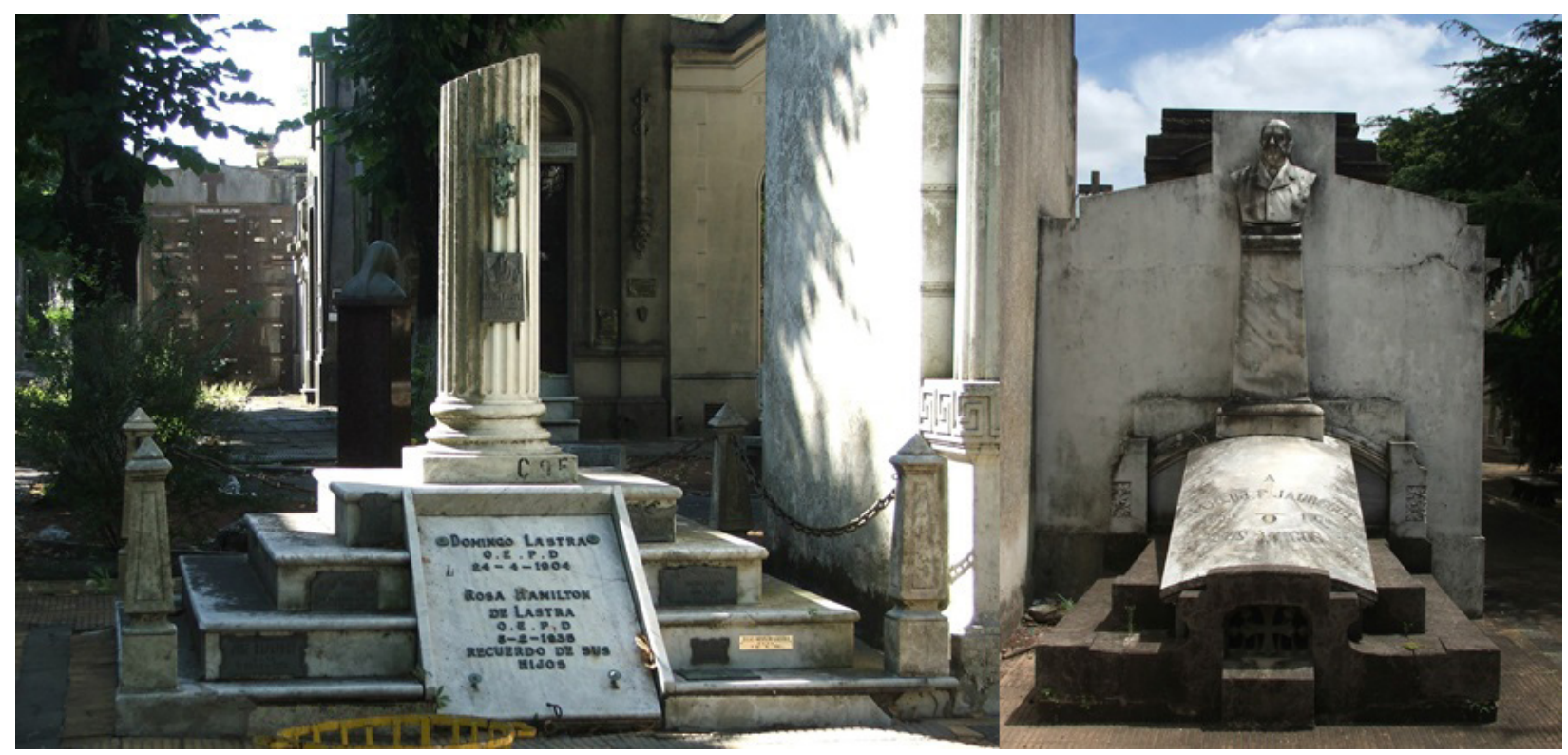

Figura 3. Izquierda: Monumento a Domingo Lastra. Derecha: Monumento y busto de Carlos Jaureguy.

\section{Análisis del área de captación de recursos}

El concepto de área de captación de recursos fue caracterizado por Vita Finzi y Higgs (1970) en sus trabajos de campo en el Monte Carmelo de Palestina. Estos autores también llamaron la atención de sus limitaciones al sostener que "cuanto más lejos del yacimiento se encuentre el área, menor será la probabilidad de ser explotado" (p.7). Otro investigador de esta problemática, Jarman (1972) sostuvo que "...Las poblaciones humanas generalmente tan solo son capaces de explotar los recursos comprendidos a una 
distancia determinada de su lugar de ocupación, sea éste un campamento, una cueva, un poblado o una ciudad" (p. 706). Con la complejización social y el surgimiento de las economías modernas de mercado, el maquinismo y la extensión de las redes camineras y ferroviarias, las áreas de captación de recursos se extendieron convirtiéndose en circuitos productivos y comerciales, temática de interés también estudiada por la Arqueología espacial (Hodder y Orton,1990).

La metodología implementada por esta disciplina, posibilita establecer el área de influencia de un lugar central como La Plata; la búsqueda de los recursos disponibles dentro del área y su caracterización. A su vez, es posible establecer la red de relaciones entre asentamientos poblados y localizaciones especializadas en actividades de explotación de los recursos disponibles y su articulación territorial. Si bien el análisis espacial ha sido criticado en su aplicación a sociedades precapitalistas arqueológicas por sus limitaciones (Criado Boado, 1993; Domínguez Berenjeno, 2001), para el caso tratado aquí, un período histórico que involucra fines del siglo XIX y principios del XX, con documentación escrita, se torna importante como concepto y como mirada. Una de las razones para su aplicación radica en que, la investigación histórica referida al proceso de federalización de la ciudad de Buenos Aires y fundación de la nueva capital provincial ha hecho hincapié en el proceso político, dejando de lado las actividades de la vida cotidiana en el proceso de urbanización de una ciudad. La necesidad de aprovisionamiento de materiales aptos para la construcción de La Plata en su etapa fundacional, por su escala implica, necesariamente un análisis del territorio local y regional para establecer la existencia o no de fuentes de aprovisionamiento de los recursos pertinentes.

\section{Circuitos productivos y áreas de aprovisionamiento de la Ciudad de La Plata.}

Los circuitos productivos y de comercialización se componen de varias etapas, en el caso de los materiales de construcción comienzan con los lugares de extracción. Éstos implican la generación de pequeñas o grandes empresas que contratan mano de obra especializada y profesionales ingenieros que organizan la extracción en base a los estudios geológicos. De acuerdo al tipo de materiales se explotan minas (de minerales metalíferos y carbón) o canteras (minerales no metalíferos) de rocas de aplicación, arenas y arcillas, generalmente a cielo abierto. El material a granel es acopiado en lugares cerrados o al aire libre en sectores específicos del área explotada, de acuerdo a las opciones tecnológicas disponibles en cada época y lugar. La segunda etapa, es la de transporte de graneles sólidos, que puede ser realizado en carros, en barcos o en tren, ya que el material se vende en toneladas. Llegado a puerto o a las plantas urbanas comienza el circuito de comercialización, mediante empresas proveedoras de materiales de construcción radicadas en las ciudades, que hacen acopio en corralones y galpones para su posterior venta a particulares.

En la memoria de los actos de gobierno de los años 1883-1884, presentada por Achaval (1885) a la Honorable Cámara de Diputados y en la reseña estadística realizada por Coni (1885) se describen los acontecimientos del festejo fundacional del 19 de noviembre de 1882. En ellas y en otros trabajos escritos sobre el acto fundacional de la ciudad de La Plata (Rey, 1932), se encuentran las primeras referencias a los materiales para construcción utilizados. Asimismo, se encuentran señaladas las rutas de aprovisionamiento de materiales locales (ladrillos y arena), regionales (el granito y la cal) desde la localidad de Azul, que para la época era la punta de riel e internacionales como Italia (mármol de carrara).

En días previos al acto de fundación el oficial albañil Antonio Ciambra junto a dos aprendices de albañil "media cuchara" y un carpintero, se dedicaron a realizar la denominada "obra negra" (Navarro, 2019), o sea la delimitación, excavación y revestimiento del hueco donde se colocaría la piedra funda- 
mental. El pozo tenía $4 \mathrm{~m}$ de largo por $3 \mathrm{~m}$ ancho y profundidad y procedieron a enladrillarlo (Leiva, 2018). El día 19 de noviembre de 1882, en el acto fundacional, se colocó en el hueco una caja de piedra de 1,15 m de largo, 0,70 cm de ancho y alto, realizada con material extraído de las canteras de Azul. En su interior tenía otra de plomo. en la cual Victorino de La Plaza puso una redoma de cristal que contenía el acta fundacional, medallas de las diferentes logias asistentes, monedas, ejemplares de las constituciones nacional y provincial, la copia del plano de la ciudad y cajas de madera conteniendo objetos realizados por la escuela de Artes y Oficios y vinos. Encima de la redoma se colocó una plancha de mármol de Carrara con la inscripción grabada: "Esta Caja contiene el acta de Inauguración de la Ciudad La Plata Capital de la Provincia de Buenos Aires". Finalmente se tapó la caja de piedra del Azul y Victorino de la Plaza la cubrió con una mezcla de cal y arena. Acto seguido, Pedro Benoit junto a los Maestros mayores de obra más antiguos de la Provincia: Miguel y Federico Cabrera, José María Baca, Juan y Agustín Rodríguez, Gregorio Almaestre y José Porret, construyeron un arco de bóveda subterráneo, cubriendo la Piedra Fundamental con ladrillos. Toda la obra involucró el uso de 12.000 ladrillos.

\section{Aprovisionamiento local}

Históricamente, desde la época colonial, el empedrado porteño se había extraído de la isla de Martín García y de canteras existentes en la costa oriental del río Uruguay. El área costera del Río de La Plata adyacente, muestra la existencia de recursos como la arena fluvial y los cordones de conchilla que permiten pensar en una explotación de cal, tal el caso de la calera jesuítica que existiera en la zona de City Bell en época colonial. La misma se corresponde con la suerte de estancia llamada Panes en el reparto de Garay, que en 1690 fue donada a los Jesuitas (Trosi Melean y Amantino. 2019). Los suelos que rodean a la ciudad de La Plata fueron explotados para la fabricación de ladrillos, permitiendo el asiento de empresas y hornos. Esta explotación generó las cavas que rodean actualmente el ejido urbano por el Norte y Oeste, en la zona del arroyo El Gato y en la localidad de Los Hornos. En 1883 funcionaban los hornos de Luis Cerrano; Luis Arditi y Rocha; Capoglio y Cía.; Bello y Bartoletti, ubicados en áreas reservadas del ejido municipal, a los que se agregó en 1905 el empresario Ctibor (Figura 4). Para 1907 en la Bolsa de Buenos Aires se registra la existencia de la Compañía Arenera del Vizcaíno como cotizante de acciones, dedicada a la explotación de estos materiales en la zona del delta del río de La Plata en ambas costas entrerriana y bonaerense (Lavandaio y Catalano, 2004), su uso aparece en las memorias descriptivas con el nombre de arena vizcaína. Sin embargo, la falta de rocas de aplicación en el entorno inmediato implicó la ampliación del área de captación de recursos más allá del medio local, plasmándose a nivel regional y transregional. 


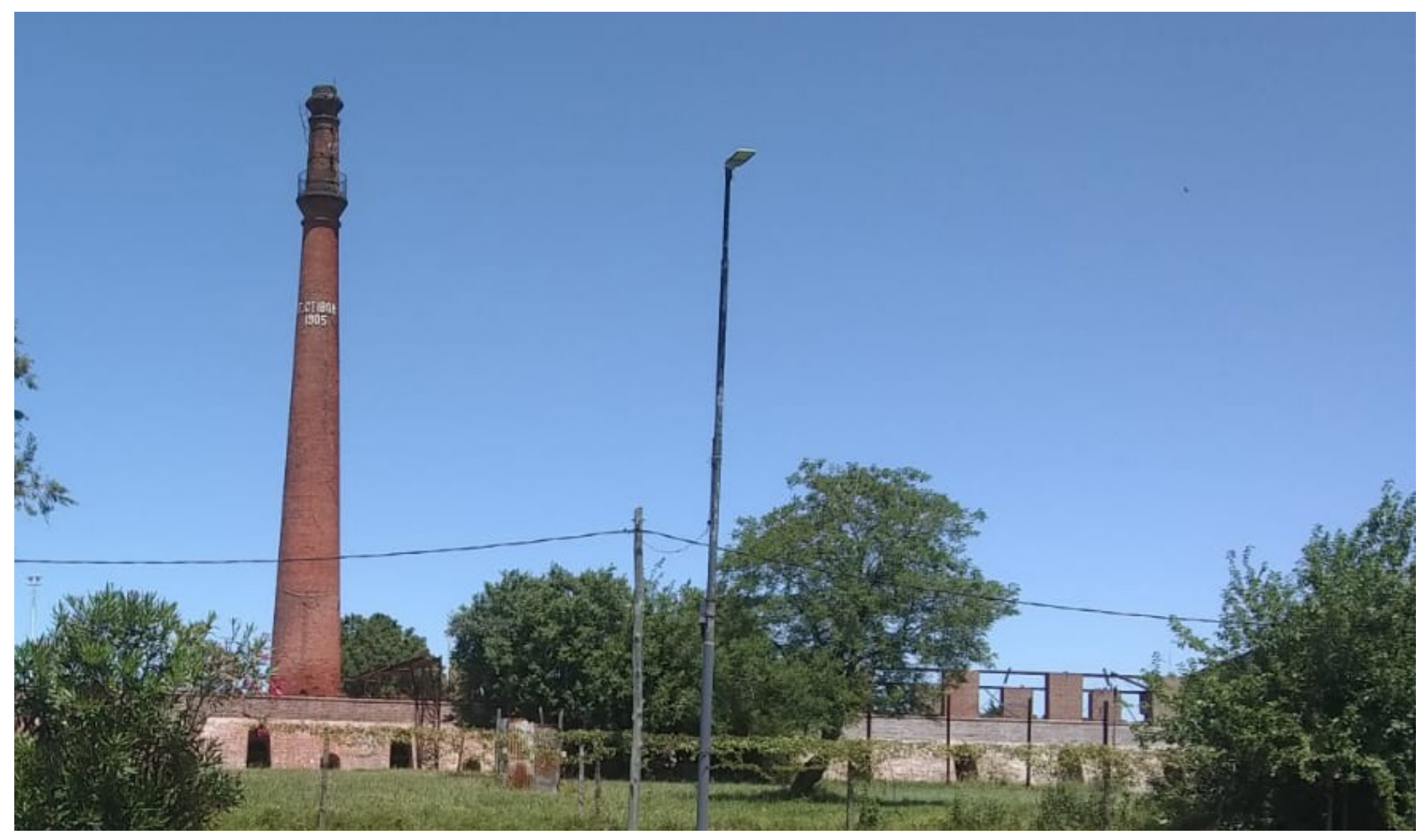

Figura 4. Chimeneas de la compañía ladrillera Ctibor actualmente en pie.

En sus adyacencias se emplaza el actual Museo del Ladrillo.

A fines del siglo XIX, la gestión política del gobierno provincial de Carlos D’Amico facilitó el establecimiento de áreas de aprovisionamiento transregionales, principalmente la región serrana bonaerense, Uruguay y Europa (Rodriguez Freire y Neira, 1885). Las primeras observaciones generales sobre explotación de materiales de uso para la construcción comienzan a fines de la época colonial con el estudio del geógrafo Miguel Lastarria (1914) realizado en 1805; quien, al referirse a la región litoraleña, caracteriza a las canteras de piedra caliza a las orillas del Uruguay como las únicas que tendría en vista la Gobernación de Buenos Aires, supliendo las necesidades de los edificios y fortificaciones.

\section{Región serrana bonaerense: las canteras y caleras}

Las investigaciones geológicas en la provincia de Buenos Aires comienzan a fines del siglo XIX con Aguirre, quien en 1879 publicó los resultados de sus trabajos de campo en sierras Bayas y su posterior estudio de la Sierra de la Tinta (1896-1897), además de su contribución sobre la Geología en el Censo provincial de 1883. A lo que se agregan los trabajos de Hauthal, investigador que recorrió y estudió las sierras bonaerenses ubicadas entre cabo Corrientes e Hinojo (1896) y las sierras de Olavarría (1901). Sin estos conocimientos, el gobierno provincial no habría podido desarrollar una política de aprovechamiento de recursos mineros específicos como las rocas de aplicación para los procesos de urbanización, ya que se consideraba que la llanura sin minerales metalíferos solo era apta para la agricultura y ganadería. En la región serrana bonaerense, de acuerdo a Angelelli et al. (1973) las relaciones geoestratigráficas eran simples, y fueron distinguidas a principios del siglo XX por Nágera (1919). La sucesión estratigráfica indica, que hay un basamento cristalino cubierto por sedimentitas de $130 \mathrm{~m}$ de espesor de los estratos de 
La Tinta; por encima sedimentos loessicos cuaternarios. En una rápida síntesis Dalla Salda et al. (2006) señalaron la importancia de los grupos serranos bonaerenses, reconociendo tres principales: Olavarría Sierras Bayas-Azul hacia el noroeste, Tandil-Barker en el sector central y Balcarce-Lobería-Mar del Plata en el extremo sudoriental. Respecto a su importancia económica se destacaron a la Formación La Tinta, “(...) formados por los horizontes de cuarcitas inferiores, dolomita, cuarcitas superiores, arcillas y calizas y material loessico. Las Sierras Australes tienen una actividad minera modesta reducida al aprovechamiento de granito, cuarcitas, arena y canto rodado." (Dalla Salda et al., 2006: 2). A fines del siglo XIX comenzaron las explotaciones de piedra caliza y granito, especialmente en el sistema de Tandilia. De acuerdo a Nario (1997) con la fundación de La Plata, el gobierno desarrolló una política de base higienista y modernización urbana, materializada en los incentivos para el adoquinamiento de calles en la nueva capital y en los pueblos de la provincia. En esta línea, en 1883 se decretó la ley de adoquinamiento, que en su artículo $2^{\circ}$ establecía que el gobierno colaboraría con el esfuerzo de las municipalidades, conduciendo gratis la piedra hasta el pueblo cuyas calles fueran a empedrarse. En el censo platense de 1884 se verifica lo expresado, pues en él se describe la utilización de adoquines de granito. Estos se asentaban sobre una capa de conchilla con un cordón exterior de piedra. Asimismo, se informa sobre la utilización de pedregullo y Pórtland y las dimensiones de las calles, bulevares y veredas (Primer Censo de Población de la ciudad de La Plata, 1884). La llegada del Ferrocarril del Sud, facilitó el transporte de piedra y la explotación de rocas de aplicación, provocando un crecimiento explosivo. Los pequeños empresarios de canteras, en su mayoría italianos expertos en la actividad, promovieron la llegada de inmigrantes especializados desde Italia, conformándose comunidades con predominio de paisanos italianos. Las explotaciones más antiguas de rocas de aplicación comenzaron hacia 1865 en Boca de la Sierra cercana a las Sierras Bayas. El pueblo homónimo se fundó en 1879 a raíz de la intensa actividad desarrollada por los primeros pioneros. Paz y Mariano (2019) señalaron la enorme importancia de la inmigración italiana en la conformación de los pueblos mineros. Un pionero italiano Ambrosio Colombo, llegado en 1860, fue el primero en explotar -con métodos preindustriales- las canteras en Sierra Chica; especialmente la dolomita para obtener cal, lo mismo ocurría en sierras Bayas. En el mercado regional de la época, se conocía el producto como "Cal del Azul"; porque el material obtenido en las canteras se llevaba en carros tirados por caballos, hasta la punta de riel de la ciudad de Azul, en un trayecto conocido con el nombre de "trayecto de la cal de azul" (Paz, 2008). La Providencia fue una explotación fundada en 1880 por Antonio Datelli y Rodolfo Avila (Paz, op cit) para producir cal viva, piedra caliza y pedregullo, que en 1885 pasó a mano de Datelli. Llegó a tener doscientos obreros, abasteciendo el mercado regional, facilitado por la inauguración en 1883 del Ferrocarril del Sud, con un ramal a la calera, posibilitándose así el aprovisionamiento de las ciudades de La Plata y Buenos Aires. Juan y Martín Gregorini explotaron el granito de Sierra Chica, en una cantera fundada en 1884 que tenía como mercado el adoquinado de Buenos Aires.

\section{Región Uruguaya}

A fines del siglo XIX y principios del XX, la explotación minera de las canteras de granito del parque Rodo en Montevideo y las del departamento La Paz fue una de las actividades económicas más importantes del Uruguay (Barrios Pintos, 1981). Estas proveían arenas de las playas del río Uruguay, granitos y mármoles de La Paz para el mercado local y la Argentina. El granito se procesaba en forma de adoquines, lozas, cordones, y grandes bloques. Para el caso, en 1854 se habían extraído veinte mil toneladas (Bra, 1990). Otro dato importante en cuanto a fuentes de aprovisionamiento es citado por Cheda Espiga (2010): 
(...) El 30 de Octubre de 1873 se lee en El Ferrocarril que el Pueblo La Paz hoy populoso es uno de los centros de población que tiene vida propia; sus magníficas canteras de piedra y de granito surten de adoquines la ciudad de Buenos Aires al extremo que no hay semana que no se exporten una asombrosa cantidad de miles de adoquines, los que dan trabajo seguro en la localidad a más de 300 obreros (...) (p.23).

La compañía inglesa C. H. Walker \& Co. Ltd., en 1880 realizó estudios en la costa uruguaya de Colonia del Sacramento para la explotación de piedra y arena y en 1887 fundó una explotación minera con máquinas, vías de tren, vagonetas y un pueblo Conchillas, para albergar a obreros calificados procedentes de Italia, Bulgaria, Grecia y uruguayos (García Díaz, 2011). Esta empresa se conformó como una factoría inglesa con moneda propia, acuñada por un ciudadano ingles Evans, que tenía almacén de ramos generales y realizaba el transporte fluvial. De las canteras de Carmelo se extraía piedra y fabricaban adoquines y cordones que llegaban mediante el transporte fluvial "La Flor del Uruguay" de la empresa Evans, a Buenos Aires y Montevideo.

El empresario uruguayo Piria tuvo una actividad de gran importancia para el desarrollo de la actividad constructiva y urbanística de Uruguay (Sempé y Gómez Llanes, 2010). En 1891 compró en Maldonado una franja de tierras que se extendía desde el cerro Pan de Azúcar hasta la costa (Floriano, s.f) comenzó la explotación de canteras produciendo setenta variedades de granito, labradorita y pórfidos del Cerro Pan de Azúcar, exportándolos principalmente a la Argentina (Baumann, 2005); para lo cual construyó un ferrocarril de $15 \mathrm{~km}$ de extensión, un puerto y el balneario de Piriápolis. Su empresa "La Central" contaba con 150 obreros (Piria, 1913). En 1887 en La Plata figura la oferta de arenas de las marcas Walker y Maldonado, provistas por el comercio de Martínez y Cía., con depósitos en la cercana Estancia Grande (Rodríguez Freire y Neira, 1887). Es importante remarcar que, en las áreas de producción de materiales de construcción, tanto de Uruguay como de Argentina, surgieron caseríos o pueblitos multiétnicos y multiculturales conformados por inmigrantes europeos y criollos que dieron origen a diversas ciudades bonaerenses como Olavarría, Tandil y Azul y uruguayas: Conchillas, Piriápolis y Minas, al igual de lo que ocurría con el poblamiento de La Plata y Montevideo.

En síntesis, las memorias constructivas de las bóvedas y panteones de la época fundacional platense dan cuenta de un amplio abanico de materiales constructivos utilizados, provenientes de diversas regiones de la provincia de Buenos Aires, y en algunos casos del exterior. Entre otros pueden mencionarse la piedra y la cal, originarias de canteras del Azul; granitos, pedregullo, cal y rocas del sistema de Tandilia y Sierras Bayas; arenas orientales de Uruguay, de río locales, así como arenas vizcaínas, dragadas de la región deltaica del Rio de La Plata. De Mar del Plata, se obtuvo la roca Balcarce, mientras que la yesería, maderas, ladrillos, marmolería, hierros, aceros y broncería, fueron de producción predominantemente local. Respecto a la roca piriapolita (pórfidos), son propias de la cantera Artigas, Uruguay. Mientras que el Cemento Blanco se obtenía por comercio desde México. Finalmente, tal como se mencionó, el mármol de Carrara, se obtenía vía trasatlántica del Norte de Italia (Figura 5). 


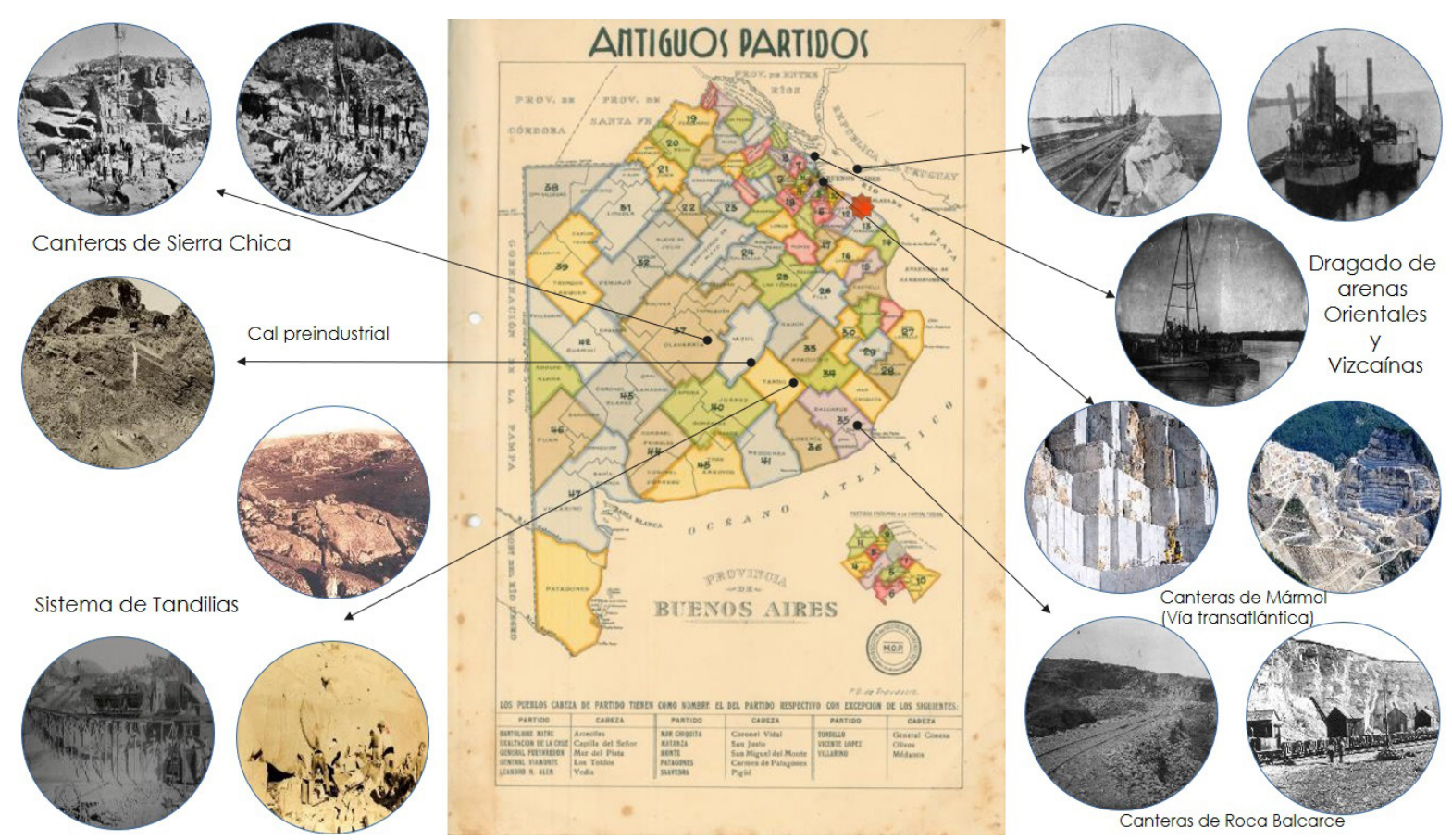

Figura 5. Localización por distrito de los materiales constructivos utilizados en la edificación de los panteones históricos del Cementerio de La Plata. Fuente: modificado de Dirección de Geodesia y Catastro de la Provincia de Buenos Aires (1940). Mapas de la Provincia de Buenos Aires (p. 4).

\section{La Comercialización local}

En la ciudad de La Plata entre 1885 y 1887 se contabilizan varios comercios de venta de materiales de construcción bajo el rubro "Comercio y materiales de edificación. Precios corrientes e Industria y Comercio de La Plata" (Rodríguez Freire y Neira, 1885).

En conjunto se contaba con 24 herrerías; 17 hojalaterías y zinguerías; 12 depósitos de venta de cal, cemento portland, tierra calcárea e hidráulica, mármoles y todo tipo de materiales de construcción; 2 fundiciones; 11 ferreterías; 90 carpinterías; 14 corralones de madera, cal y "fierro", una sola broncería y 5 aserraderos (Sempé y Baldini, 2011).

En algunos casos como Cerrano y Cía., traía cal viva y apagada de Paraná, Córdoba y Azul; de esta última localidad proviene la piedra cuadrada de granito para adoquines y cordones de veredas. El corralón de Santiago Carbone y Cía., proveía maderas de "Nord-América" y Paraguay, piedras hamburguesas para veredas y hierro galvanizado. Nazario Zapararte y Cía., Rizzi y Cía., traían piedra para cordones desde Azul y Gregorio Gallino arena oriental, al igual que Martínez y Cía., que también proveía de arenas de las firmas Walker y de Maldonado, en Uruguay. Todo lo cual redundó en el afianzamiento del circuito de producción y comercialización respecto a la capital provincial para fines del siglo XIX, garantizando el aprovisionamiento de estos materiales en una ciudad que estaba en pleno proceso de urbanización. Asimismo, se aseguraron estas actividades mediante las políticas del gobierno Provincial que propiciaban el transporte gratuito de adoquines durante el gobierno de Rocha y el posterior Decreto del Poder Ejecutivo (1885) firmado por D'Amico y Achaval “disponiendo que la Oficina de Delineaciones sólo 
dará la línea para las construcciones de material” (p. 315). Otra acción importante fue la reorganización funcional de la municipalidad platense en 1908 con la creación de la oficina de Obras Públicas ocupada de los permisos de obras para edificaciones, refacciones, excavaciones, construcción de cercos y veredas, entre otros; y la compilación de todas las normas referidas a la edificación tanto de obras públicas como privadas, publicada en el Digesto Municipal de ese año (MLP, 1908). La existencia de varias firmas de agentes marítimos consignatarios como Molino Torres y Cía., Manuel Gómez y Cía., G.L. D’Amico y Cía., Márquez y Elena corredores que se encargaron de la carga y descarga de buques, aseguraron también el transporte de materiales de procedencia fluvial y europea (Rodríguez y Neira, 1885 y1887).

\section{Reflexión final}

Se considera que la mirada involucrada desde la antropología es dual, como arqueología histórica realiza una arqueología del documento, en busca de datos aislados y un trabajo de campo que atestigüe los datos registrados. Desde una antropología histórica va en búsqueda de significaciones, permitiendo establecer relaciones entre hechos. Sin la conformación de una sociedad multicultural durante la última mitad del siglo XIX -que cambió la estructura social argentina, sus costumbres, ideas y concepción del mundo- la fundación de la ciudad de La Plata no hubiera sido posible. La conformación multiétnica y multicultural en la estructura de su comunidad urbana tiene su réplica en los pequeños pueblos surgidos en las explotaciones mineras de la provincia de Buenos Aires y de Uruguay, que conformaron su área de aprovisionamiento de recursos.

En este trabajo, la documentación de base es la originada en la edificación construida en el espacio funerario: los expedientes de obras particulares con sus memorias constructivas, que nos permitieron establecer y demarcar las actividades realizadas en la cotidianeidad de una comunidad urbana fundacional y la articulación entre los diferentes agentes sociales relacionados al campo económico comercial e industrial de la época. A través del estudio de la materialidad del campo funerario fue posible reconstruir las redes comerciales para el aprovisionamiento urbano y establecer cuáles fueron las áreas de captación de recursos en la etapa fundacional y así también comprobar que, a falta de insumos locales, se produjo la expansión y creación de nuevas redes de aprovisionamiento. Destacamos que los procesos de aprovisionamiento de los materiales constructivos fueron los mismos para el binomio Ciudad-Cementerio. En este sentido, afirmamos la existencia de una doble condicionalidad a diferentes niveles: lo local y lo regional; por un lado la Ciudad y su Cementerio con correspondencia entre procesos constructivos y materias primas usadas; por el otro, a mayor escala, entre La Plata y las regiones extra locales de aprovisionamiento de materiales. Retomando el concepto de Proyecto a Gran Escala y teniendo en mente su limitada temporalidad, cobra mayor significación el valor de dichos centros o focos de aprovisionamiento.

En el proceso de investigación sobre la obtención de materiales de construcción, se reconoció y registró la expansión y creación de redes de aprovisionamiento. El uso de los mármoles de procedencia europea, en especial las variedades italianas Carrara, boticcino y negro usados a fines del siglo XIX y principios del XX, en algunos casos traídos como lastre por los migrantes con oficios especializados. Posteriormente fue más frecuente el uso de granitos nacionales o uruguayos por la facilidad de aprovisionamiento regional de rocas de aplicación debido a la política estatal de tendido de vías de comunicación ferroviarias, abaratando los costos de construcción y el valor de las materias primas. Cabe señalar que, debido al circuito de producción y comercialización, afianzado en el siglo XIX, estuvo asegurado el aprovisionamiento de la ingente cantidad de materiales necesarios para la construcción de una ciudad y su cementerio. Como así también, la existencia de varias firmas de agentes marítimos consignatarios que 
aseguraron el transporte de materiales de procedencia europea.

Finalmente, es importante marcar que la demanda masiva de materias primas, a regiones como Tandil, Olavarría, Azul, Maldonado, entre otros, contribuyó, sin dudas, al desarrollo multiétnico de estas localidades, por la radicación de mano de obra especializada extranjera -evidenciado en su crecimiento demográfico no vegetativo coetáneo- similar al fenómeno registrado en La Plata. De este modo, se incrementó una retroalimentación positiva entre el Centro (capital provincial) y su Periferia tendiente a maximizar sus mutuos desarrollos.

\section{Referencias bibliográficas}

Angelelli V., Villa J. R. y Suriano, J. M. (1973). Recursos Minerales y Rocas de Aplicación de la Provincia de Buenos Aires. Anales LEMIT. https://digital.cic.gba.gob.ar/handle/11746/912

Baumann, N. (2005) Breve historia de la explotación de sienita en Piriápolis (Departamento de Maldonado), Uruguay. Revista Sociedad Uruguaya de Geología SUG, (12), 4-15.

Barrios Pintos, A. (1981). Canelones. Su proyección en la historia nacional (Tomo II). Intendencia Municipal de Canelones.

Bra, G. (1990). El Crecimiento de Buenos Aires. Producción, transporte y Comercialización de arena y piedra entre 1850 y 1950. Todo es Historia, 273, 41 -57.

Barba, D.E. (1998). La Plata en sus primeros años de vida. Revista El Museo, (12), 49-54.

Catullo, M. R. (1998). Poder y Participación en Proyectos de Gran Escala. Análisis comparativo de los procesos de relocalización por la construcción de la represa binacional argentino- uruguaya de Salto Grande. [Tesis de Doctorado, Universidad de Brasilia].

Catullo, M. R. (2015). La Ciudad de La Plata como Primer Proyecto de Gran Escala: su contexto socioeconómico. Cadernos do Tempo Presente, 27-39.

Carrasquero, S. I., Adam, S. y Sempé, M. C. (2012). El uso del mármol como expresión de la memoria: algunos ejemplos en los cementerios de La Plata, Azul y Juárez. II encuentro sobre antroposemiótica de la muerte y el morir: dialogismo(s) transdisciplinar(es). Facultad de humanidades y ciencias sociales. Universidad Nacional de Misiones. Posadas. Misiones. Argentina.

Cheda Espiga, R. D. (2010). Los Picapedreros de La Paz 1872 - 1928. Ministerio de Educación y Cultura, Dirección Nacional de Cultura Uruguay.

Criado-Boado, F. (1993). Límites y posibilidades de la arqueología del paisaje. Revista de Arqueología y Prehistoria, 2, 9-55.

Dalla Salda L., Spalletti, L., Poiré, D., De Barrio, R., Echeveste, H. y Benialgo, A. (2006). Tandilia. Temas de la Geología Argentina I INSUGEO. Serie Correlación Geológica, (21), 17-46.

Delledonne, M., Massola, P. y Corbalán, A. (2011). Estructura administrativa y ordenanzas municipales del cementerio de La Plata. En M. C. Sempé y O. Flores (comp) El Cementerio de La Plata y su contexto histórico (pp. 63-97). Ed. El Autor. 
Domínguez Berenjeno, E. L. (2001). Arqueología y territorio: de la" interpretación arqueológica" al" dato histórico". SPAL, 10, 109-122.

Fava F. (2019). Liberar tiempo para inventar el tiempo. En Paz y Mariano (comp.) Inmigrantes Italianos en las canteras de Sierra Chica Tandil._https://www.soc.unicen.edu.ar/images/editorial/ebooks/ paz2019.pdf

Floriano Jorge (s.f). Francisco Piria. El Hombre y su Obra.http://www.autoresdeluruguay.uy/biblioteca/ Francisco_Piria/lib/exe/fetch.php?media=piria-_jorge_floriano.pdf

García Díaz L. E. (2011). Conchillas. Memorias de un médico rural. Editorial Trilce.

Hodder I y C. Orton (1990). Análisis espacial en arqueología. Crítica.

Jarman M.R. (1972). A territorial model for archaeology: a behavioural and geographical approach. En D.L. Clarke (ed.) Models in Archaeology (pp.705-734). Methuen.

Lavandaio E. y E. Catalano (2004). Historia de la minería Argentina. Anales 40, Instituto de Geología y Recursos Minerales SEGEMAR.

Leiva A. D. (2018). La trastienda del acto fundacional de la ciudad de la plata (19 de noviembre de 1882) Instituto de Estudios Históricos de San Fernando de Buena Vista.

https:/historiasanfernando.com.ar/la-trastienda-del-acto-fundacional-de-la-ciudad-de-la-plata-19-denoviembre-de-1882/

Nágera, J. (1919). La Sierra Baya, estudio geológico y económico. Anales. Sección geología, mineralogía y minería. Editor Talleres Gráficos del Ministerio de Agricultura de la Nación.

Nario H. (1997). Los Picapedreros. Tandil. Historia abierta 2. Ediciones del Manantial.

Navarro J. (2019). ¿Qué es obra negra, obra gris y obra blanca?.https://www.admagazine.com/arquitectura/diferencia-entre-obra-negra-obra-gris-y-obra-blanca-en-cosntruccion-20191028-6082-articulos.html

Paz C. A. (2008). La Producción de cal protoindustrial en las sierras de Olavarría. Butlletí D’Arqueología Industrial i de Museus de Ciencia i Técnica, 1988-2008.

Paz C. A. y C. Mariano (2019). Inmigrantes italianos en las canteras de Sierra Chica. Tandil. Universidad Nacional del Centro de la Provincia de Buenos Aires.

https://www.soc.unicen.edu.ar/images/editorial/ebooks/paz2019.pdf

Piria, F. (1913). Riquezas desconocidas del Uruguay. Tipografía El Arte.

Rey J. M. (1932). La Nueva Capital, Los Primeros Cinco Años de su Construcción. Ed. J. Peuser.

Ribeiro, G. L. (1987). ¿Cuánto más grande mejor? Proyectos de Gran Escala: una forma de producción vinculada a la expansión de sistemas económicos. Desarrollo Económico, 27 (105), 3-28.

Rodríguez Freire M. y Neira, J. (1885). Índice: Materiales de construcción y precios corrientes. Guía 
Revista mensual de La Plata.

Rodríguez Freire, M. y Neira, J. (1887). Índice: Industria y Comercio. Guía Revista mensual de La Plata, 27.

Sempé M. C. y Baldini, M. I. (2011). La Plata y su etapa fundacional. En M. C. Sempé y O. Flores (comp) El Cementerio de La Plata y su contexto histórico (pp. 36-57). Ed. El Autor.

Salas, C. P. y Condomi Alcorta, A. (1910). Censo general de la ciudad de La Plata, capital de la provincia. Población, propiedad raíz, comercio é industrias, levantado en los días 22 al 30 de mayo de 1909. Talleres "La Popular".

Sempé M. C. y Gómez Llanes, E. (2010). La tumba del Alquimista: La Bóveda Piria. Imagens Da Morte Anais Eletrônicos do IV Congresso Latino-americano de Ciências Sociais e Humanidades. Niteroi Brasil

Troisi Melean J. C. y Amantino M. (2019) Jesuitas en las Américas. Presencia en el tiempo. Ex Libris TeseoPress.

Vita-Finzi, C. y Higgs, E. (1970). Prehistory economy in the Mount Carmel Area of Palestine:site catchment analysis. Proceedings of the Prehistoric Society, 36: 1-37.

\section{Fuentes consultadas}

Achával N. (1885). Memoria presentada por el ministro secretario en el Departamento de Gobierno; Dr. D. Nicolás Achaval a la H. Legislatura de la Provincia. Años 1883 - 84. La Plata.

Aguirre, E. (1879). La geología de la sierra Baya. Anales de la Sociedad Cientifica Argentina, 8, 34-45. Aguirre, E. (1896-1897). Notas Geológicas sobre la sierra de la Tinta. Anales Museo nacional de Buenos Aires, 5, 333-397.

Aguirre, E. (1883). Constitución Geológica. Censo General de la provincia de Buenos Aires, (pp.22-39). Archivo General de la Nación, Mapoteca II-117.

Censo General de la Provincia de Buenos Aires 1883. Realizado el 9 de Octubre de 1881. Bajo la administración del Dr. Don Dardo Rocha 1883. Imprenta de El Diario, Buenos Aires.

Coni, E. R. (1885). Reseña estadística y descriptiva de La Plata, capital de la provincia de Buenos Aires, Buenos Aires, Ministerio de Gobierno, 1885.

Dirección de Geodesia y Catastro de la Provincia de Buenos Aires (1940). Mapas de la Provincia de Buenos Aires, (p.4).

Hauthal, R. (1896). Contribución al estudio de la geología de la Provincia de Buenos Aires. Revista del Museo de La Plata, 8, 477-484.

Hauthal, R. (1901). Apuntes geológicos de las sierras de Olavarría. Contribuciones al Conocimiento de la geología de la provincia de Buenos Aires. Publicaciones de la Universidad de La Plata. Facultad de Ciencias Físico Matemáticas II, (1), 17-30.

Lastarria, M. (1914) (1805). Colonias Orientales del Río Paraguay o de La Plata. Documentos para la historia argentina. Compañía Sudamericana de Billetes de Banco.

MLP (1908). Municipalidad de La Plata. Digesto Municipal. Compilado por el señor Comisionado doctor Alfredo C. Paz por decreto del 17 de Mayo de 1907. Taller de Impresiones Oficiales. 
MLP (1885). Decreto del Poder Ejecutivo, disponiendo que la Oficina de Delineaciones sólo dará la línea para las construcciones de material, 24 de enero de 1885. Firmado D’Amico-Nicolás Achaval. MLP 1902 Oficina técnica, trámites internos, 1902. Permiso de construcción Panteón A 15-24 MLP (1902) Oficina técnica, trámites internos, 1902. Permiso de construcción Panteón A 22MLP MLP (1906) Oficina técnica, trámites internos, 1906. Permiso de construcción Panteón A 40 MLP, (1908) Oficina técnica, trámites internos, 1908. Permiso de construcción Panteón A 26 MLP 1908 Oficina técnica, trámites internos, 1908. Permiso de construcción Panteón A 52 Primer Censo de Población de la ciudad de La Plata. (1884). Oficina de Estadística, La Plata.

Recibido: 3 de marzo de 2021

Aceptado: 14 de julio de 2021 